\title{
Optofluidically Tunable Multimode Interference Erbium Doped Fiber Laser
}

\author{
J. E. Antonio-Lopez ${ }^{1}$, I. Hernandez-Romano ${ }^{1}$, D. A. May-Arrioja ${ }^{2}$, J. J. Sanchez-Mondragon ${ }^{1}$, and P. LiKamWa ${ }^{3}$ \\ ${ }^{1}$ Photonics and Optical Physics Laboratory, Optics Department, INAOE \\ Apdo. Postal 51 y 216, Tonantzintla, Puebla 72000, México \\ ${ }^{2}$ Depto. de Ingeniería Electrónica, UAM Reynosa Rodhe, Universidad Autónoma de Tamaulipas, \\ Carr. Reynosa-San Fernando S/N, Reynosa, Tamaulipas 88779, México. \\ ${ }^{3}$ CREOL and FPCE, The College of Optics and Photonics, University of Central Florida, Orlando, FL 32816-2700
}

\begin{abstract}
A stable optofluidically tunable fiber laser based on Multimode Interference (MMI) effect is experimentally demonstrated. The tuning mechanism relies on modifying the effective width of the multimode fiber (MMF) using a liquid with a specific refractive index, which in turns tunes the peak wavelength of the filter. We easily demonstrate a tunability of almost $40 \mathrm{~nm}$ with a side-mode suppression ratio (SMSR) of 47 $\mathrm{dBm}$, and a $3 \mathrm{~dB}$ bandwidth of $0.4 \mathrm{~nm}$. The laser was operated at room temperature without any thermal control and the wavelength peak was very stable.
\end{abstract}

\section{INTRODUCTION}

Study of MMI effects started on planar waveguides and has $N_{\text {been intensively applied in the development of beam }}$ splitter, combiners, and multiplexers for optical communications [1]. Nowadays, the MMI effects in fibers are being widely investigated and also its applications towards the development of different photonic devices [2-4]. Using such structure a very sensitive sensors with No-Core fiber in SMFMMF-SMF structures was demonstrated [5], as well as the demonstration of a very simple and cost-effective tunable fiber lasers [6]. In the case of the tunable laser, we exploited the fact that by increasing the effective length of the MMI device the peak wavelength can be modified. However, there is always the limitation of having moving fibers, as well as issues with the uniformity of the diameter of the capillary used for this tunable MMI laser. A simple and elegant solution to this issue is to alter the MMF characteristics without moving any fiber at all. In this work we demonstrate the tuning of a MMI device using a fluid with a specific refractive index. The idea behind this mechanism is to modify the cladding of a NoCore MMF when liquid is added to it. The effect of the liquid is to increase the effective width of the No-Core fiber and this will shift the peak wavelength of the MMI device. We show a simple mechanism to obtain a linear and continuos tuning mechanism. Using this novel optofluidically tunable MMI filter a tunable laser is demonstrated with a tuning range of almost $40 \mathrm{~nm}$ going from $1534 \mathrm{~nm}$ to $1572 \mathrm{~nm}$, with a very high stability in all the unable range.

\section{MULTIMODE INTERFERENCE FIBER FILTER}

Based on the Fig. 1, the operation of the MMI filter can be explained as follows. The only requirement is a MMF that supports several modes $(\geq 3)$, which is spliced between two SMF's. After the supported modes are excited by launching a field using the input SMF, the interference between the modes propagating along the MMF gives rise to the formation of selfimages of the input field along the MMF. Therefore the length of the MMF has to be precisely cleaved in order to have a selfimage right at the facet of the output SMF. The MMI effect has been previously studied and the length of the MMF can be calculated using:

$$
L=P \frac{3}{4} L \quad \text { with } \quad L=\frac{4 n_{M M F} D_{M M F}^{2}}{3_{0}}
$$

where $P$ corresponds to the self-image number $(P=0,1,2, \ldots)$ and $L_{\pi}$ is the beat length, here $n_{M M F}$ and $D_{M M F}$, are the refractive index and effective width of the MMF respectively and $\lambda_{0}$ as free space wavelength. According to Eq. 1, selfimages should be periodically formed along the MMF. However, since the fourth image exhibits minimum losses, the MMI filter was operated at the fourth image.

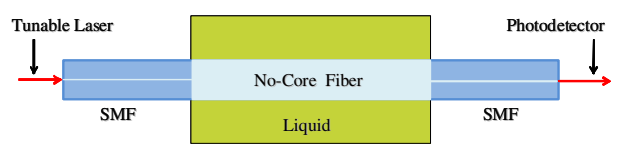

Fig. 1. Schematic the tuning mechanism for the tunable MMI fiber filter.

\section{OPTOFLUIDIC TUNABLE LASER DESIGN}

\section{A).TUNABLE FILTER DESIGN}

To make this a tunable filter, we have to look at the wavelength dependence of the filter. By combining the MMI governing Eq. (1) and expressing the peak wavelength in terms of all the other parameters we obtain:

$$
{ }_{0}=P \frac{n_{M M F} D_{M M F}^{2}}{L}
$$


as shown in Eq. (2), in order to tune the peak wavelength response of the filter, we need to modify the refractive index, the length, or the diameter of the MMF, so modifying the MMF diameter would give us the biggest tuning change due to its quadratic dependence. The fact that our cover is air, it means that we can modify the fiber properties if the cladding region is modified, so when the index contrast between core and cladding is reduced, the effective diameter (fundamental mode width) of the No-Core fiber is increased, so, the liquid level is raised ( $n=1.434$ in this case) and starts to cover the No-Core fiber, the resulting effect is similar to having two different MMF with slight different diameters. Therefore, the required phase factor will now be determined by the contribution of these two MMF's, as stated in Eq. (3). The first part in the sum is the contribution of the segment of Nocore fiber with liquid around, and the second part of the sum is the contribution of the No-Core fiber without liquid. Here $L_{t}$ is the total No-Core fiber length, $L_{n}$ is the No-core fiber length with liquid, $D_{M M F n}$ is the new diameter for the No-core fiber length with liquid (as calculated from the Goes-Hanchën equation), $L_{0}$ is the No-core fiber length without liquid, and $D_{M M F O}$ is the original diameter of the No-core fiber $(125 \mu \mathrm{m})$. Depending on the amount of No-Core fiber filled with liquid, the peak wavelength can be easily calculated. Wavelength tuning of the MMI filter is achieved as show in the fig. 2.

$$
{ }_{0}=4 \frac{n_{M M F} D_{M M F n}^{2}}{L_{t}}\left(L_{n}\right)+4 \frac{n_{M M F} D_{M M F 0}^{2}}{L_{t}}\left(L_{0}\right)
$$

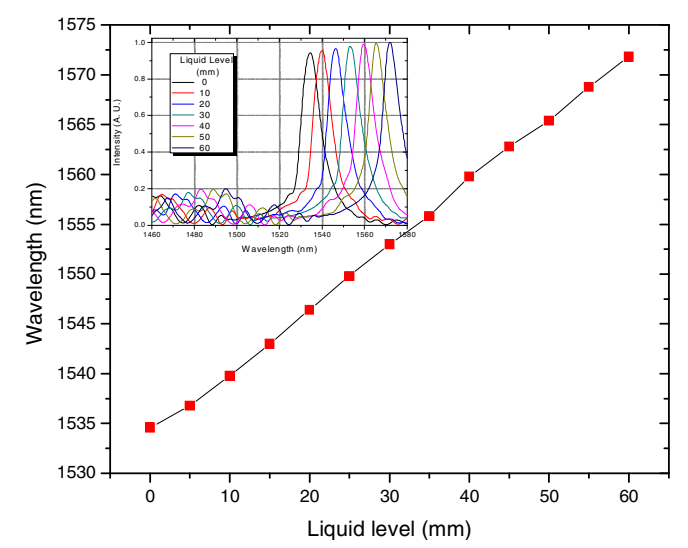

Fig. 2. Peak wavelength response against liquid level with $5 \mathrm{~mm}$ increments Inset: wavelength response of the MMI filter when the liquid level is raised every $10 \mathrm{~mm}$.

\section{B). - TUNABLE LASER DESIGN}

The cavity laser was composed of a 980/1550 nm WDM, a polarization-independent isolator, our optofluidically tunable filter, and a directional coupler with a splitting ratio of 10:90. The gain medium in the cavity was a $5 \mathrm{~m}$ long piece of EDF used in the experiment with a doping level corresponding to absorption coefficients of 3.8 and $1.5 \mathrm{~dB} / \mathrm{m}$ at $980 \mathrm{~nm}$, respectively. The pump power was provided by a $980 \mathrm{~nm}$ laser diode from the $980 \mathrm{~nm}$ port of WDM. The output laser was coupled out from the $10 \%$ port of the coupler and measured with an Aguilent optical spectrum analyzer (OSA). The super imposed spectral response of the tunable laser is shown in Fig. 4.

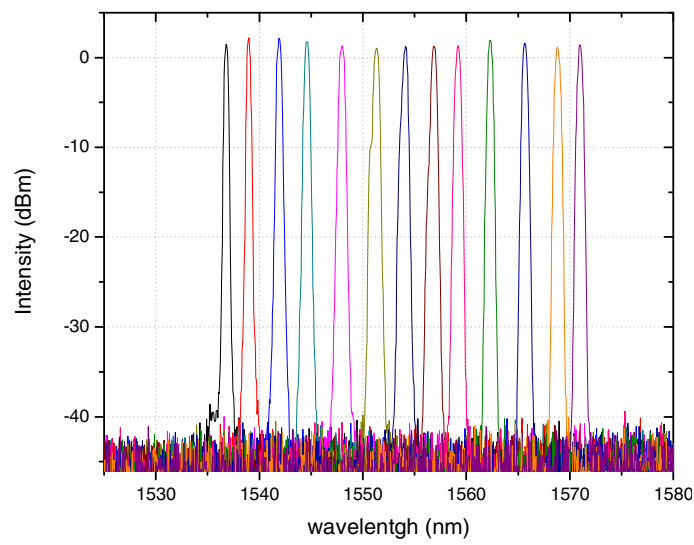

Fig. 3. Superimposed spectral response of the tunable MMI fiber laser.

We can see that the wavelength tuning is continuous over the whole tuning range and follows direct changes of the liquid level along the No-Core fiber. A SMSR of $47 \mathrm{~dB}$ was also achieved, with a $3-\mathrm{dB}$ bandwidth of $0.4 \mathrm{~nm}$. Power variation is also minimum across the tuning range, which eliminates the need for a variable attenuator.

\section{CONCLUSIONS}

A novel optofluidic tuning mechanism was proposed using MMI effects in fibers. We demonstrated that the fabrication processes for this tunable filter is quite simple and cost effective. Using this optofluidically tunable MMI fiber filter a tunable laser was demonstrated with a linear tuning range of almost $40 \mathrm{~nm}$ that easily covers the C-band of the EDF with very good stability, good efficiency and signal noise ratio.

\section{REFERENCES}

[1] Lucas B. Soldano and E. C. M. Pennings, "Optical multi-mode interference devices based on self-imaging: principles and applications", J. Lightwave Technol., 13(4), 615-627 (1995).

[2] Q. Wang, G. Farrell and W. Yan, "Investigation on Single-ModeMultimode-Single-Mode Fiber structure", J. Ligthwave Technol., 26(5), 512-519 (2008).

[3] X. Zhu, A. Schülzgen, H. Li, L. Li, L. Han, J. V. Moloney and N. Peyghambarian, "Detailed investigation on self-imaging in large-core multimode optical fibers for application in fiber lasers and amplifiers", OPTICS EXPRESS, 16(21), 16632-16645 (2008).

[4] W. S. Mohammed and P. W. E. Smith, Xijia Gu, "All-fiber multimode interference bandpass filter”, Opt. Lett., 31(17), 2547-2549 (2006).

[5] J. E. Antonio-Lopez, D. Lopez-Cortes, M. A. Basurto-Pensado, D. A. May-Arrioja and J. J. Sanchez-Mondragon, "All-Fiber Multimode Interference Refractometer Sensor", Proc. of SPIE 7316, 73161F-173161F-5, (2009).

[6] J. E. Antonio-Lopez, A. Castillo-Guzman. D. A. May-Arrioja, R. Selvas-Aguilar and P. LiKamWa, "Tunable Multimode Interference Bandpass Fiber Filter”, Opt. Lett. 35 (3) 324-326 (2010). 